\title{
Worry Is Good for Breast Cancer Screening: A Study of Female Relatives from the Ontario Site of the Breast Cancer Family Registry
}

\author{
Li Rita Zhang, ${ }^{1}$ Anna M. Chiarelli, ${ }^{2,3}$ Gord Glendon,, 4 Lucia Mirea, ${ }^{3,5}$ Julia A. Knight, ${ }^{1,3}$ \\ Irene L. Andrulis, ${ }^{1,4}, 6$ and Paul Ritvo ${ }^{2,7}$ \\ ${ }^{1}$ Samuel Lunenfeld Research Institute, Mount Sinai Hospital, Toronto, ON, Canada M5T 3L9 \\ ${ }^{2}$ Prevention and Cancer Control, Cancer Care Ontario, Toronto, ON, Canada M5G 2L7 \\ ${ }^{3}$ Dalla Lana School of Public Health, University of Toronto, Toronto, ON, Canada M5T 3M7 \\ ${ }^{4}$ Ontario Cancer Genetics Network, Samuel Lunenfeld Research Institute, Mount Sinai Hospital, Toronto, ON, Canada M5G 1 X5 \\ ${ }^{5}$ Maternal-Infant Care Research Centre, Mount Sinai Hospital, Toronto, ON, Canada M5G 1X6 \\ ${ }^{6}$ Department of Molecular Genetics, University of Toronto, Toronto, ON, Canada M5G 1A8 \\ ${ }^{7}$ School of Kinesiology and Health Sciences, York University, Toronto, ON, Canada M3J 1P3
}

Correspondence should be addressed to Li Rita Zhang, rita.zhang@lunenfeld.ca

Received 22 February 2012; Accepted 27 April 2012

Academic Editor: T. L. Vaughan

Copyright ( 2012 Li Rita Zhang et al. This is an open access article distributed under the Creative Commons Attribution License, which permits unrestricted use, distribution, and reproduction in any medium, provided the original work is properly cited.

\begin{abstract}
Background. Few prospective studies have examined associations between breast cancer worry and screening behaviours in women with elevated breast cancer risks based on family history. Methods. This study included 901 high familial risk women, aged 23-71 years, from the Ontario site of the Breast Cancer Family Registry. Self-reported breast screening behaviours at year-one followup were compared between women at low $(N=305)$, medium $(N=433)$, and high $(N=163)$ levels of baseline breast cancer worry using logistic regression. Nonlinear relationships were assessed using likelihood ratio tests. Results. A significant non-linear inverted " $U$ " relationship was observed between breast cancer worry and mammography screening $(P=0.034)$ for all women, where women at either low or high worry levels were less likely than those at medium to have a screening mammogram. A similar significant non-linear inverted " $U$ " relationship was also found among all women and women at low familial risk for worry and screening clinical breast examinations (CBEs). Conclusions. Medium levels of cancer worries predicted higher rates of screening mammography and CBE among high-risk women.
\end{abstract}

\section{Introduction}

In 2010, about 8,900 Ontario women were diagnosed with breast cancer while 2,100 died of the disease [1]. Compared to women without a family history of breast cancer, women with an affected first-degree relative are about twice as likely to develop breast cancer, with risks increasing when any firstdegree relatives are diagnosed prior to age 50, and when larger numbers of first-degree relatives are diagnosed [2, 3]. A recent review indicated that screening mammography reduced breast cancer mortality for women 39-69 years [4], and while no differentiation was found by familial breast cancer risk, other studies support the effectiveness of mammography and clinical breast examination (CBE) in breast cancer detection in elevated risk women [5, 6]. During the study time period, the Canadian Task Force on Preventive Health Care recommended breast screening by mammography and $\mathrm{CBE}$ every 1-2 years for average risk women aged 50-69 years [7]. For women with elevated familial risk, mammogram, CBE, and breast self-examinations (BSE) [8] are recommended annually and prior to 50 years of age [9$11]$.

Worry is a central construct in theories concerning preventive health behaviours like breast cancer screening, and the Self-Regulation theory suggests that, depending on conditions, worry may impede or advance disease management [12]. In an earlier paper, Hailey hypothesized that the inverted U-shaped curve best portrayed associations 
between anxiety or fear and screening behaviours in contrast to linear associations [13]. Building on self-regulation theory and the Hailey hypothesis, the cognitive-social health information processing (C-SHIP) model proposed a "curvilinear" or "inverted U" relationship between worry intensity and screening adherence. According to this model, negative feelings about the self are frequently activated during health information processing, and, once activated, high levels of negative effect and anxious arousal promote avoidance, while lower levels lead to reduced motivation, such that very low and very high levels are associated with lower screening adherence [14], and middle levels are associated with higher adherence levels.

Extensive research has been conducted examining the association of worry and breast cancer screening behaviours in average risk women [15-17]. In particular, the metaanalysis of prospective studies by Hay et al. [16] supports a positive influence of breast cancer worry on screening behaviours (mammography and BSE). However, since these studies included women at average risk, most exhibited a narrow range of mild worry, it precluded the examination of potential nonlinear associations.

Other observational studies on women at elevated familial breast cancer risk found inconsistent associations between worry and breast cancer screening adherence. The majority have examined associations between current worry levels and past breast screening behavior, and, in two of the crosssectional studies, no significant associations were found between cancer-related worry and mammography utilization $[18,19]$, while a third reported an inverse association between higher levels of mammography screening adherence and lower levels of breast cancer worry [20]. But in other types of screening, namely, CBE and breast ultrasound use, no associations were found between higher uptake levels and higher worry levels $[18,19]$.

Only two prospective studies examined the association between breast cancer worry and breast screening in high familial risk women $[21,22]$, and, in one, cancer worry at moderate levels significantly predicted greater mammography adherence [21], while in the other women with higher levels of breast cancer worry that impacted mood were significantly less likely to utilize mammography than those with low worry levels [22].

To further examine the association between worry and breast screening and to identify potential nonlinear associations, our study prospectively followed a population-based cohort of women at high familial risk. We examined associations between baseline worries of developing breast cancer and screening behaviours at one-year followup for these women with at least one first-degree relative diagnosed with breast and/or ovarian cancer. Breast screening adherence behaviours were compared between women at low, medium, and high breast cancer worry levels according to familial risk.

\section{Methods}

2.1. Study Population. This study identified a cohort of female relatives of incident invasive breast cancer cases from the Ontario site of the Breast Cancer Family Registry (BCFR) funded by the United States National Cancer Institute. The details of the BCFR and the Ontario site of the BCFR have been previously described [23]. Invasive breast cancer cases (probands), pathologically confirmed and diagnosed between 1996 and 1998, were identified from the Ontario Cancer Registry. Physicians were contacted to obtain permission to mail patients a cancer Family History Questionnaire (FHQ). Respondents meeting defined family history criteria and a random sample $(25 \%)$ of those not meeting criteria were asked to participate in the Ontario BCFR site. Of those eligible $(N=2587), 1851(72 \%)$ probands participated.

These probands were asked for address information and for permission to contact relatives (first degree, those affected with breast, ovarian, or certain other cancers, and their first degree relatives). An invitation letter to participate in the Ontario BCFR site was sent to relatives, and those who agreed to participate were sent an Epidemiology Questionnaire (EQ) between 1998 and 2004. This study was conducted a few years after initial recruitment of relatives and included all female relatives enrolled in the Ontario BCFR site who completed an $E Q$ between 20 and 69 years of age and who were unaffected by breast cancer at the time of the proband's diagnosis date. From the 3374 participating female relatives, 1514 were residents of Ontario and met study criteria.

Of the 1514 women who were sent a Personal History and Screening Questionnaire (PHSQ) between November 2005 and March 2007, 1314 (86.8\%) were contacted, and $1114(84.8 \%)$ consented to interview. Upon exclusion of the 37 with a breast cancer diagnosis, the Year One Followup Personal History and Screening Questionnaire (Y1FPHSQ) was sent to 1077 women approximately one year following the PHSQ interview. Of the 1049 (97.6\%) women contacted and eligible, 965 (92.0\%) agreed to participate. Participants who only had a second-degree relative with breast cancer $(N=30)$, who had bilateral mastectomy $(N=6)$, or who had a breast cancer diagnosis since the PHSQ $(N=6)$ were excluded. Since women at elevated familial breast cancer risk are recommended to have annual breast screening, the 22 women who completed the Y1FPHSQ more than 548 days (1.5 years) of completing the PHSQ were also excluded. Thus, the final study cohort consisted of 901 women. This study was approved by the Research Ethic Boards of Mount Sinai Hospital and the University Health Network.

2.2. Data Collection. Information was obtained from three questionnaires. The first $(E Q)$ was self-administered during recruitment of female relatives into the Ontario BCFR site; it collected detailed information on demographics and key behavioural risk factors for breast and ovarian cancers. The two subsequent questionnaires (PHSQ and Y1FPHSQ) of similar contents were telephone administered, and they updated changes in health behaviours and key demographic characteristics from the $E Q$ questionnaire and collected detailed information on breast cancer screening examinations and breast cancer worries. Eligible participants were sent an introductory letter with a copy of the PHSQ or the Y1FPHSQ approximately two weeks prior to contact by 
phone. This allowed time for participants to recall specific dates and events and allowed reference to the questionnaire during the interview; further clarification was provided by the trained telephone interviewer during the interview process when necessary. More details of the questionnaire instruments have been previously published [23-25].

Overall baseline worry of developing breast cancer was assessed by two questions on the PHSQ adopted from earlier work by Lerman et al. [26, 27]. Each question had choice options of "not at all or rarely," "sometimes," "often," and "almost all the time." The questions were (1) "During the past month, how often have you thought about your own chances of developing breast cancer?" and (2) "During the past month, how often have you worried about your own chances of developing breast cancer?" Participants were considered to have low levels of breast cancer worry if responding "not at all or rarely" to both questions, a medium level if answering "sometimes" to either or both questions, and a high level if responding "often" or "almost all the time" to either or both questions.

The PHSQ (baseline assessment) also asked if participants had a mammogram, CBE, or breast ultrasound since EQ completion. The Y1FPHSQ asked if participants had these examinations since completing the PHSQ. Both questionnaires asked participants whether the main reason for having these examinations was for screening or for a nonscreening purpose (e.g. due to a "breast problem or symptom," "followup of a previous breast problem," or "participation in a research study"). For each screening test in the prospective analysis, participants were dichotomized into those who had a test at year one followup and those who did not. Women who had these examinations for nonscreening purposes were excluded for analyses involving the specific test. Self-reported date of last mammogram was validated against medical records to assess recall accuracy. Age at interview at baseline was calculated as the difference in years between date of birth and date of PHSQ interview. Descriptive analyses employed age categories, but regression models were adjusted using continuous age. The highest level of education attained at baseline was obtained from the corresponding question on the PHSQ. Body mass index in $\mathrm{kg} / \mathrm{m}^{2}$ at baseline $(<25,25$ to $<30$, and $\geq 30$ ) of the participants was derived from information on height (EQ) and weight (PHSQ). The average annual frequency of visiting a health care facility in the past two years (once a year or less, 2 to 3 times a year, and 4 or more times a year) was determined using responses to the PHSQ. Both the EQ and PHSQ assessed prior benign breast diseases. A positive history of benign breast disease was defined as a positive response to either or both questionnaires, whereas negative responses to both questionnaires were considered as absence of benign breast disease history. Perceived risk of developing breast cancer (much below or below average, same average risk, above average, much above average) was determined using the PHSQ question adopted from a comparative measure used earlier by Lipkus et al. [28], "Compared to other women your age, how likely are you to get breast cancer in your lifetime?"
Classification of family history of breast and/or ovarian cancer was based on information collected from the FHQ completed by the relatives' proband using a modified definition of previously referenced groups for familial breast cancer risk [5, 9]. Table 1 shows the criteria for classifying women to low, moderate, or high familial risk to breast and/or ovarian cancer.

2.3. Statistical Methods. Chi-square tests assessed the association of worries of developing breast cancer (categorized as high, medium, or low) with each demographic or personal characteristic. The internal consistency between the two questions that assessed baseline worry was estimated using Chronbach's alpha. Multivariate logistic regression examined relationships between breast cancer worry assessed at baseline and breast cancer screening behaviours at yearone followup. For each screening behavior, models were fit using medium worry as the reference group. Final models were adjusted for age, education, annual frequency of visiting a health care facility, perceived breast cancer risk, the corresponding baseline screening behavior, and familial risk. As familial risk was identified as a potential effect modifier in the model between baseline worry and mammogram screening at followup, further analyses stratified by familial breast cancer risk were also conducted. Since many study participants were related and might share common cancer screening behaviours, potential correlation due to family clustering was corrected by using robust error variances in all regression models [29]. To test for nonlinear associations between breast cancer worry and screening behaviours, likelihood ratio tests compared the model with worry defined as a continuous $(0,1,2)$ variable to the model with worry defined by a categorical (low, medium, and high) variable. Due to stratification and linearity assessment, a large number of statistical tests were performed; however, as these did not examine independent association hypotheses (even the two primary outcomes mammography and CBE are strongly associated, Chi-square $P<0.0001$ ), a multiple testing correction was not applied. All statistical analyses were conducted using SAS version 9.1 [30], and significance of all statistical tests was evaluated using two-sided $P$ values at the $5 \%$ level.

\section{Results}

The analysis cohort included 901 women aged 23-71 years from 593 unique families of which 395 (67\%) had one family member, 122 (20\%) had 2 family members, and 76 $(13 \%)$ had 3 and up to 6 family members. Chronbach's alpha, which measured the internal consistency between the two questions that assessed baseline worry, was 0.85 . Of the women interviewed, 305 (34\%) reported a low level of worry, $433(48 \%)$ reported a medium level, and $163(18 \%)$ reported a high level under our overall worry level categorization (Table 2). Women with either medium or high levels of worry compared to low were significantly more likely to be less than 60 years of age, to have visited a health care 
TABLE 1: Classification of familial risk to breast and/or ovarian cancer.

\begin{tabular}{ll}
\hline Familial risk group & Family history of breast and/or ovarian cancer \\
\hline High & Two or more first-degree relatives with breast and/or ovarian cancer diagnosed at any age \\
& One or more first-degree relative(s) with both breast and ovarian cancer diagnosed at any age \\
& One or more first-degree relative(s) diagnosed with bilateral breast cancer at any age \\
& A personal history of ovarian cancer \\
\hline Moderate & A self-reported Ashkenazi Jewish background \\
& One first-degree relative with breast cancer diagnosed before age of 40 \\
& One first-degree relative with ovarian cancer \\
& One first-degree relative with breast cancer diagnosed after the age of 40 and two or more second-degree relatives \\
& with breast cancer diagnosed at any age \\
\hline Low & One first-degree relative diagnosed with breast cancer after the age of 40 \\
\hline
\end{tabular}

TABLE 2: Distribution of demographic and personal characteristics according to worry for female relatives from the Ontario site of the Breast Cancer Family Registry.

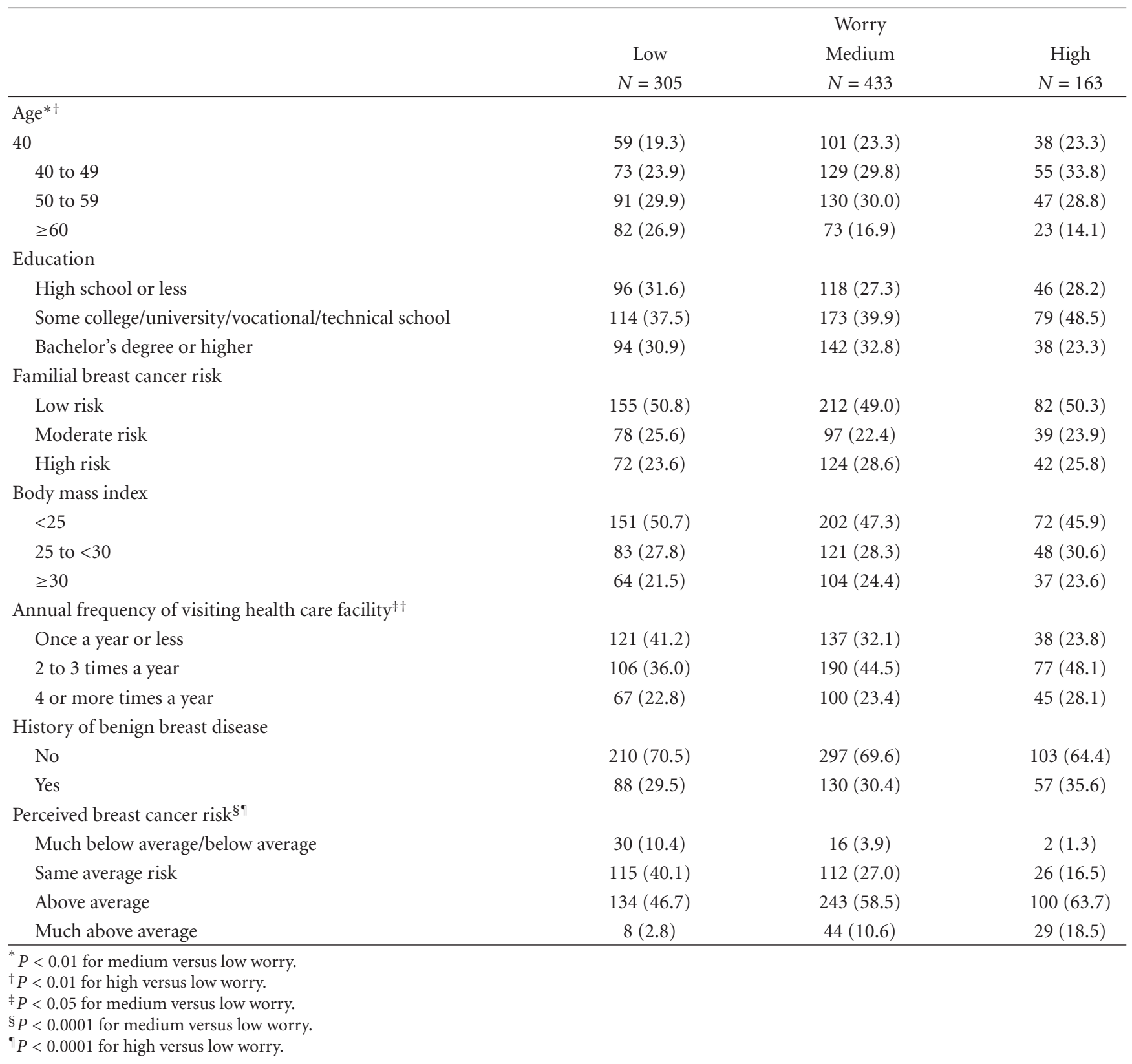


facility at least twice annually and to perceive their breast cancer risk as above or much above average compared to other women their age (Table 2). There were no significant differences between the three worry levels within strata defined by educational level, familial breast cancer risk, body mass index, or history of benign breast disease.

At year-one followup (Table 3), a significant nonlinear relationship was observed between breast cancer worry and mammography screening in all women $(P$ value $=$ $0.034)$. Compared to all women at a medium worry level of developing breast cancer, women at low worry were significantly less likely to have a screening mammogram (OR : 0.66, 95\% CI : 0.45-0.98). Consistent with an inverted U-shaped relationship, all women with high compared to medium worry were less likely to have a mammogram although this association was not significant (Table 3 and Figure 1(a)). Although not significant, a similar inverted Ushaped relationship was observed for women at moderate or high familial risk. Compared to those with medium worry levels, women with low worry (OR : $0.52,95 \% \mathrm{CI}: 0.30-0.90$ ) and with high worry levels (OR:0.77, 95\% CI:0.41-1.44) were less likely to have a screening mammogram.

For all women and for women at low familial risk, those at high worry levels were significantly less likely than those with medium worry to have CBE screening at yearone followup (OR: $0.52,95 \%$ CI : 0.34-0.82; OR : 0.40, 95\% CI : 0.21-0.76, resp.). In both groups, a significant nonlinear inverted U-shaped relationship was observed between worry and CBE (Table 3 and Figure 1(b)).

Compared to those with medium worry, all women with high levels of worry were more likely to have a screening breast ultrasound at year one followup (OR: 1.90, 95\% CI : 1.00-3.62) although this relationship did not reach significance. A borderline significant nonlinear relationship $(P$-value $=0.051)$ was observed for women at moderate or high familial breast cancer risk. These women were more likely to have a screening breast ultrasound if their breast cancer worry was low or high compared to medium (low versus medium OR: 1.76, 95\% CI: 0.78 - 3.96; high vs. medium OR:2.36, 95\% CI:0.91-6.10) although these associations were not significant. In contrast, women at low familial risk were less likely to have a breast ultrasound if their worry was low compared to medium $(\mathrm{OR}=0.53 ; 95 \%$ CI : 0.19-1.46) (Table 3 and Figure 1(c)).

\section{Discussion}

Among all women in this cohort, we observed a significant nonlinear inverted " $U$ " relationship indicating a lower likelihood of obtaining a screening mammogram at followup if baseline worry levels were low or high compared to medium. A similar and significant inverted " $U$ " association was found between worry and CBE for all women and women at low familial risk.

Our prospective analyses are consistent with the cognitive-social health information processing model, as medium worry levels predicted the likelihood of screening mammogram uptake at one year followup in all women.
A similar conclusion was noted in two other prospective studies. In one, moderate cancer worry levels significantly associated with greater mammography adherence at oneyear followup [21], while in the other, the odds of obtaining a mammogram at 12 months were $70 \%$ lower for women reporting a higher level of worry that impacted mood, compared to lower worry levels [22].

Our study is the first to examine the relationship between breast cancer worry and CBE utilization prospectively, and to find a significant inverted U-shaped association for all women and women at low familial risk. Women were less likely to have a screening CBE at year-one followup if worry was low or high compared to medium. As previous crosssectional studies reported no significant association between breast cancer worry and CBE utilization $[18,19]$, it is likely that differences in study design accounted for these discrepant results. The reverse influence of screening results on worry would be of greater concern in a cross-sectional examination as compared to our prospective analysis.

We found higher but insignificant breast ultrasound uptake for women regardless of familial risk level at high cancer worry compared to medium worry; this finding is consistent with a previous cross-sectional study where no significant associations between worry and breast ultrasound examination were reported [19]. As breast ultrasound is usually recommended as a supplement to mammography in breast cancer screening in women with higher familial risk, worry levels may play a smaller role on its uptake in comparison to those of mammography or CBE. This could explain the differences in the associations observed between mammogram and CBE versus breast ultrasound.

The present study had notable strengths; a large cohort of female relatives of breast cancer cases from a populationbased registry were studied with adequate power to examine all associations. To-date, our study is the first to examine prospectively the associations between breast cancer worry and $\mathrm{CBE}$ and breast ultrasound, in addition to mammography. Our analysis was stratified by degree of familial breast cancer risk, after a significant interaction between worry and familial risk was observed for mammography screening uptake at followup. In other studies, women with family histories of breast cancer exhibited a higher level of worry than women without $[31,32]$, and higher familial risk influenced breast cancer screening practices [33, 34].

Attempts were made to reduce study limitations by minimizing misclassifications through the use of selfreported breast screening behaviours. Although self-reported mammography data has been found to be accurate for determining whether a woman had a mammogram, selfreported data is less accurate in determining the duration since last mammogram [35] since women tend to underestimate resulting in overestimations of recent mammography use $[36,37]$. To minimize recall inaccuracy, the PHSQ and YIFPHSQ were mailed to the participants to allow recollection of dates and events prior to telephone interview. To estimate the magnitude of recall bias, self-reported date of last mammogram was validated against medical records, and approximately $92 \%$ of women reported their last mammogram to be within 12 months of the actual date. 


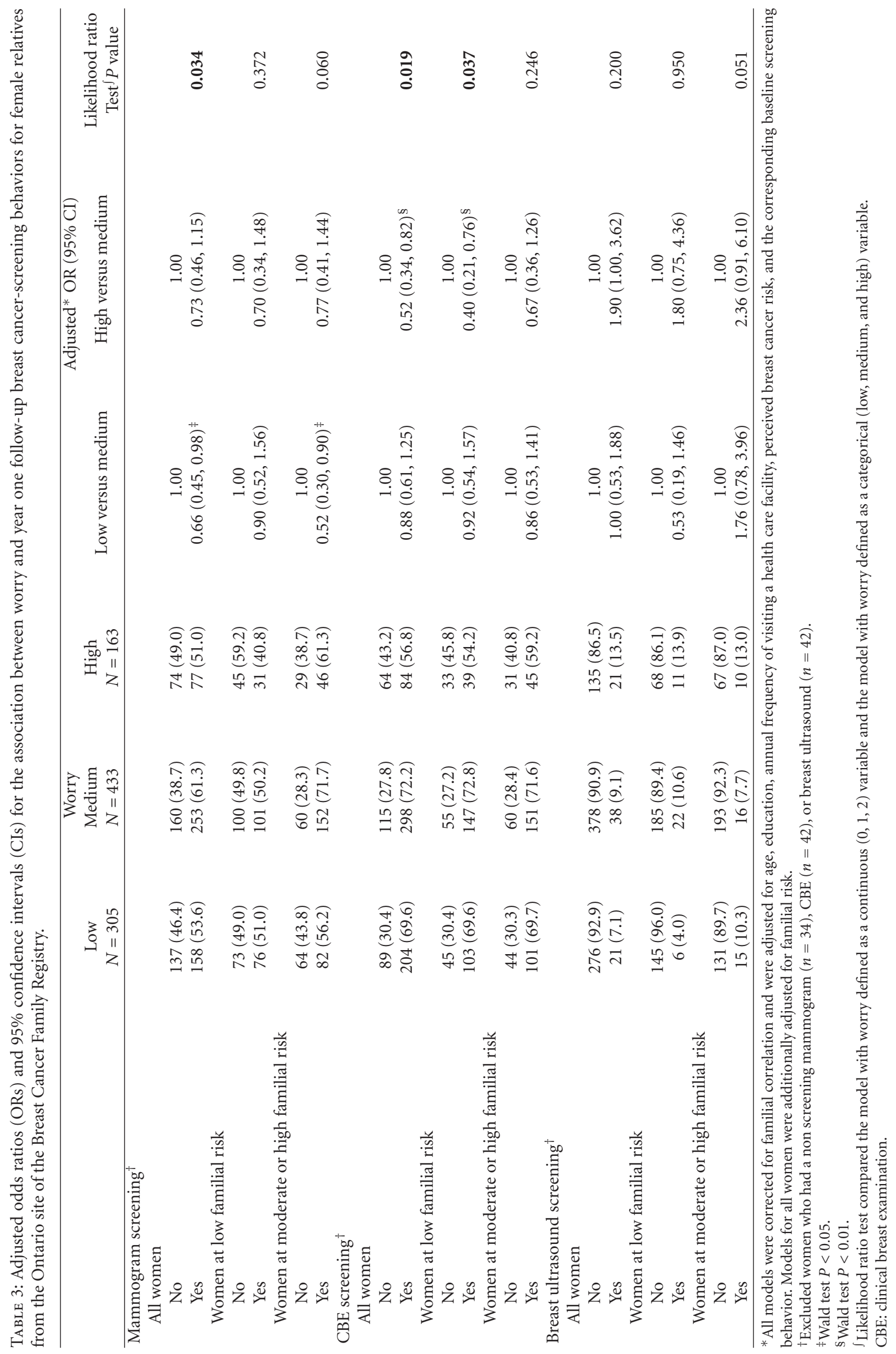




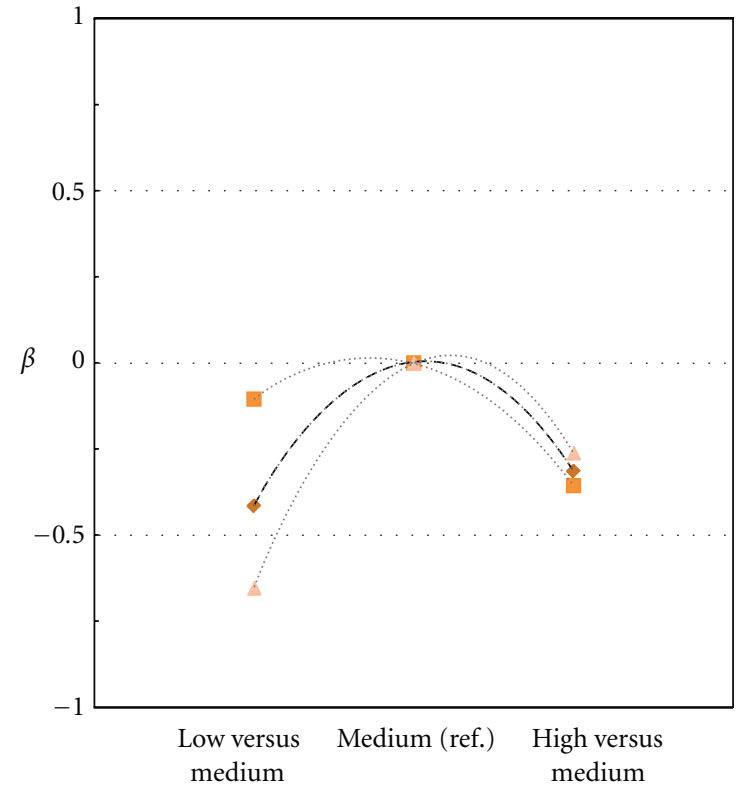

(a)

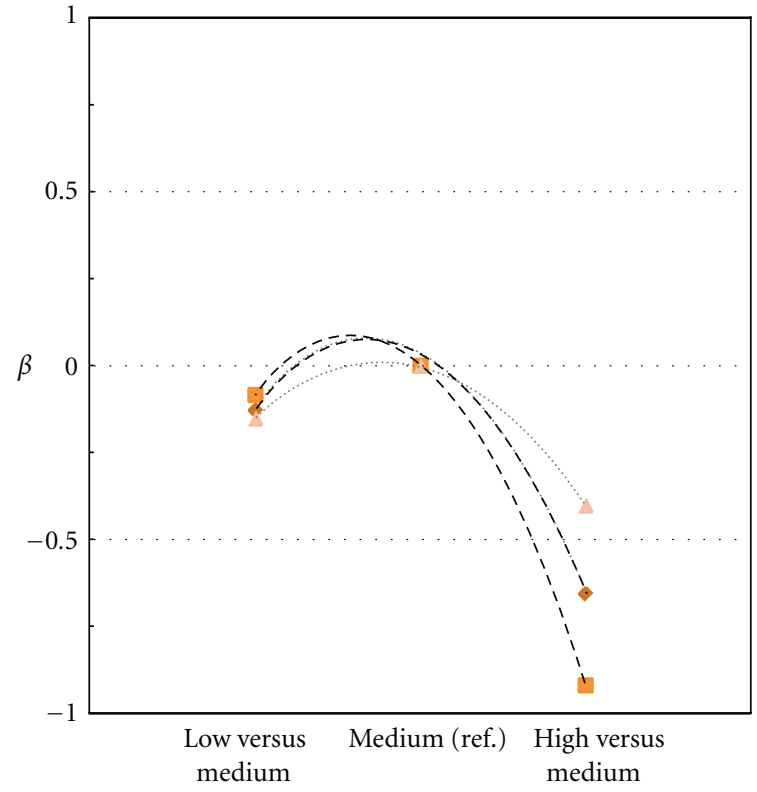

(b)

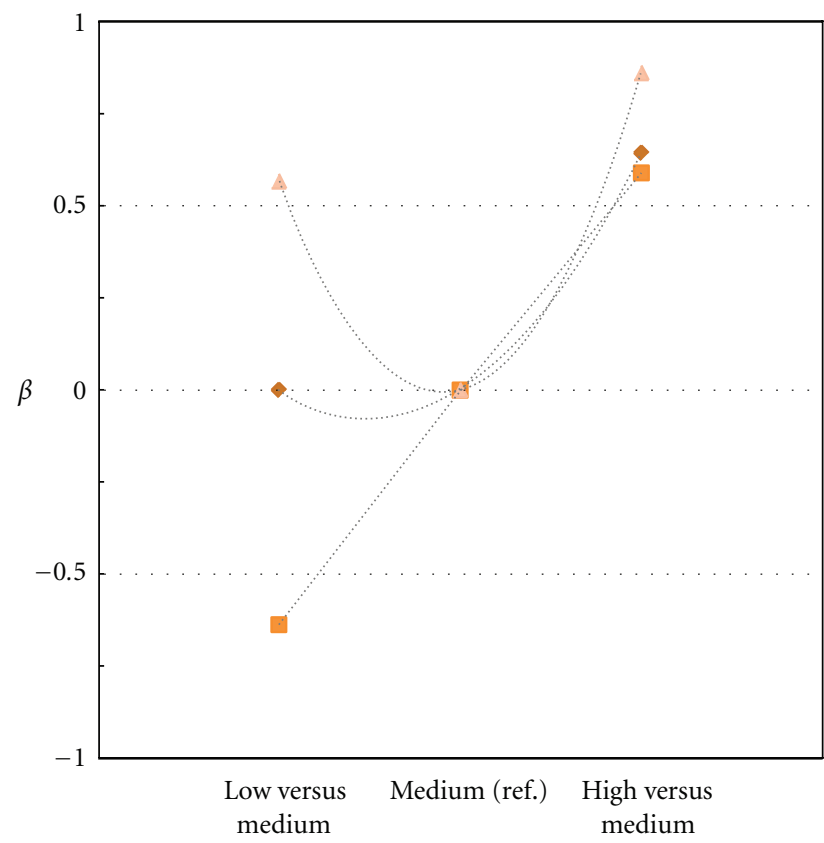

(c)

Figure 1: Adjusted parameter estimates $(\beta \mathrm{s})$ for the association between worry and year-one followup breast screening behaviors for female relatives from the Ontario site of the Breast Cancer Family Registry. (a) Mammogram; (b) clinical breast examination; (c) breast ultrasound examination. (Diamonds: all women; squares: women at low familial risk; triangles: women at moderate/high familial risk; dark trendlines: likelihood ratio test $P$ value $<0.05)$.

Participants in this study were residents of Ontario with universal health care coverage and an organized breast cancer screening program for women 50 years of age and older. Therefore, findings may be less applicable to populations where there is more limited health care coverage or access to screening.

In this cohort of women at elevated familial risk for breast cancer, medium levels of worry were more influential than either low or high worry levels for the uptake of screening mammography and CBE. Furthermore, the strength of association between worry and screening behaviours depended on the level of familial risk. Findings from this study are important for clinical management and development of educational materials that address worry levels for women with various levels of familial breast cancer risk in order to achieve optimal breast cancer screening adherence. 


\section{Conflict of Interests}

The authors declare that there is no conflict of interests.

\section{Acknowledgments}

The authors thank the study staff: Nada Abdel-Malek, Elizabeth Arbour, Kristine Cooper, Sabiha Faruqui, Jennifer Kohn, Elaine Maloney, Lindsay Stewart, and Nayana Weerasooriya. They also thank the participants in the Ontario site of the Breast Cancer Family Registry. This research was supported by the Canadian Breast Cancer Research Alliance (Grant 016270). This work was also supported by the National Cancer Institute, National Institutes of Health under RFA-CA-06-503, and through cooperative agreements with members of the Breast Cancer Family Registry and Principal Investigators, including Cancer Care Ontario (U01 CA69467). The content of this manuscript does not necessarily reflect the views or policies of the National Cancer Institute or any of the collaborating centers in the Breast CFR, nor does mention of trade names, commercial products, or organizations imply endorsement by the US Government or the Breast CFR.

\section{References}

[1] Canadian Cancer Society's Steering Committee, Canadian Cancer Statistics 2011, Canadian Cancer Society, Toronto, Canada, 2011.

[2] Collaborative Group on Hormonal Factors in Breast Cancer, "Familial breast cancer: collaborative reanalysis of individual data from 52 epidemiological studies including 58, 209 women with breast cancer and 101, 986 women without the disease," The Lancet, vol. 358, no. 9291, pp. 1389-1399, 2001.

[3] P. D. Pharoah, N. E. Day, S. Duffy, D. F. Easton, and B. A. Ponder, "Family history and the risk of breast cancer: a systematic review and meta-analysis," International Journal of Cancer, vol. 71, no. 5, pp. 800-809, 1997.

[4] H. D. Nelson, K. Tyne, A. Naik, C. Bougatsos, B. K. Chan, and L. Humphrey, "Screening for breast cancer: an update for the U.S. Preventive Services Task Force," Annals of Internal Medicine, vol. 151, no. 10, pp. 727-737, 2009.

[5] L. Cortesi, D. Turchetti, I. Marchi et al., "Breast cancer screening in women at increased risk according to different family histories: an update of the Modena study group experience," BMC Cancer, vol. 6, article 210, 2006.

[6] E. E. Halapy, A. M. Chiarelli, N. Klar, and J. Knight, "Breast screening outcomes in women with and without a family history of breast and/or ovarian cancer," Journal of Medical Screening, vol. 11, no. 1, pp. 32-38, 2004.

[7] B. J. Morrison, "Screening for breast cancer," in Canadian Task Force on Periodic Health Examination. Canadian Guide to Clinical Preventive Health Care, pp. 788-795, Health Canada, Ontario, Canada, 1994.

[8] M. U. Yood, B. D. McCarthy, N. C. Lee, G. Jacobsen, and C. C. Johnson, "Patterns and characteristics of repeat mammography among women 50 years and older," Cancer Epidemiology Biomarkers and Prevention, vol. 8, no. 7, pp. 595599, 1999.

[9] D. M. Eccles, D. G. R. Evans, and J. Mackay, "Guidelines for a genetic risk based approach to advising women with a family history of breast cancer," Journal of Medical Genetics, vol. 37, no. 3, pp. 203-209, 2000.

[10] D. G. R. Evans and F. Lalloo, "Risk assessment and management of high risk familial breast cancer," Journal of Medical Genetics, vol. 39, no. 12, pp. 865-871, 2002.

[11] E. Warner, R. E. Heisey, V. Goel, J. C. Carroll, and D. R. McCready, "Hereditary breast cancer: risk assessment of patients with a family history of breast cancer," Canadian Family Physician, vol. 45, pp. 104-112, 1999.

[12] H. Leventhal, M. Diefenbach, and E. A. Leventhal, "Illness cognition: using common sense to understand treatment adherence and affect cognition interactions," Cognitive Therapy and Research, vol. 16, no. 2, pp. 143-163, 1992.

[13] B. J. Hailey, "Family history of breast cancer and screening behavior: an inverted U-shaped curve?" Medical Hypotheses, vol. 36, no. 4, pp. 397-403, 1991.

[14] S. M. Miller, Y. Shoda, and K. Hurley, "Applying cognitivesocial theory to health-protective behavior: breast selfexamination in cancer screening," Psychological Bulletin, vol. 119, no. 1, pp. 70-94, 1996.

[15] J. L. Hay, T. R. Buckley, and J. S. Ostroff, "The role of cancer worry in cancer screening: a theoretical and empirical review of the literature," Psycho-Oncology, vol. 14, no. 7, pp. 517-534, 2005.

[16] J. L. Hay, K. D. McCaul, and R. E. Magnan, "Does worry about breast cancer predict screening behaviors? A meta-analysis of the prospective evidence," Preventive Medicine, vol. 42, no. 6, pp. 401-408, 2006.

[17] N. S. Consedine, C. Magai, Y. S. Krivoshekova, L. Ryzewicz, and A. I. Neugut, "Fear, anxiety, worry, and breast cancer screening behavior: a critical review," Cancer Epidemiology Biomarkers and Prevention, vol. 13, no. 4, pp. 501-510, 2004.

[18] C. Foster, D. G. R. Evans, R. Eeles et al., "Predictive testing for BRCA 1/2: attributes, risk perception and management in a multi-centre clinical cohort," British Journal of Cancer, vol. 86, no. 8, pp. 1209-1216, 2002.

[19] C. Isaacs, B. N. Peshkin, M. Schwartz, T. A. DeMarco, D. Main, and C. Lerman, "Breast and ovarian cancer screening practices in healthy women with a strong family history of breast or ovarian cancer," Breast Cancer Research and Treatment, vol. 71, no. 2, pp. 103-112, 2002.

[20] C. Lerman, M. Daly, C. Sands et al., "Mammography adherence and psychological distress among women at risk for breast cancer," Journal of the National Cancer Institute, vol. 85, no. 13, pp. 1074-1080, 1993.

[21] M. A. Diefenbach, S. M. Miller, and M. B. Daly, "Specific worry about breast cancer predicts mammography use in women at risk for breast and ovarian cancer," Health Psychology, vol. 18, no. 5, pp. 532-536, 1999.

[22] M. D. Schwartz, K. L. Taylor, and K. S. Willard, "Prospective association between distress and mammography utilization among women with a family history of breast cancer," Journal of Behavioral Medicine, vol. 26, no. 2, pp. 105-117, 2003.

[23] E. M. John, J. L. Hopper, J. C. Beck et al., “The breast cancer family registry: an infrastructure for cooperative multinational, interdisciplinary and translational studies of the genetic epidemiology of breast cancer," Breast Cancer Research, vol. 6, no. 4, pp. R375-R389, 2004.

[24] M. A. Campitelli, A. M. Chiarelli, L. Mirea et al., "Adherence to breast and ovarian cancer screening recommendations for female relatives from the Ontario site of the breast cancer family registry," European Journal of Cancer Prevention, vol. 20, no. 6, pp. 492-500, 2011. 
[25] L. R. Zhang, A. M. Chiarelli, G. Glendon et al., "Influence of perceived breast cancer risk on screening behaviors of female relatives from the Ontario site of the breast cancer family registry," European Journal of Cancer Prevention, vol. 20, no. 4, pp. 255-262, 2011.

[26] C. Lerman, B. Trock, B. K. Rimer, C. Jepson, D. Brody, and A. Boyce, "Psychological side effects of breast cancer screening," Health Psychology, vol. 10, no. 4, pp. 259-267, 1991.

[27] C. Lerman, B. Trock, B. K. Rimer, A. Boyce, C. Jepson, and P. F. Engstrom, "Psychological and behavioral implications of abnormal mammograms," Annals of Internal Medicine, vol. 114, no. 8, pp. 657-661, 1991.

[28] I. M. Lipkus, M. Kuchibhatla, C. M. McBride et al., "Relationships among breast cancer perceived absolute risk, comparative risk, and worries," Cancer Epidemiology Biomarkers and Prevention, vol. 9, no. 9, pp. 973-975, 2000.

[29] G. Zou, "A modified poisson regression approach to prospective studies with binary data," American Journal of Epidemiology, vol. 159, no. 7, pp. 702-706, 2004.

[30] SAS Institute Incocporation, Statistical Analysis Software 9.2, SAS Institute, Cary, NC, USA, 2008.

[31] M. Cohen, "Breast canceer early detection, health beliefs, and cancer worries in randomly selected women with and without a family history of breast cancer," Psycho-Oncology, vol. 15, no. 10, pp. 873-883, 2006.

[32] F. Gil, I. Méndez, A. Sirgo, G. Llort, I. Blanco, and H. CortésFunes, "Perception of breast cancer risk and surveillance behaviours of women with family history of breast cancer: a brief report on a Spanish cohort," Psycho-Oncology, vol. 12, no. 8, pp. 821-827, 2003.

[33] L. Madlensky, R. A. Vierkant, C. M. Vachon et al., "Preventive health behaviors and familial breast cancer," Cancer Epidemiology Biomarkers and Prevention, vol. 14, no. 10, pp. 2340-2345, 2005.

[34] B. Meiser, P. Butow, A. Barratt et al., "Breast cancer screening uptake in women at increased risk of developing hereditary breast cancer," Breast Cancer Research and Treatment, vol. 59, no. 2, pp. 101-111, 2000.

[35] Q. Yang, M. J. Khoury, C. Rodriguez, E. E. Galle, L. M. Tatham, and W. Dana Flanders, "Family history score as a predictor of breast cancer mortality: prospective data from the cancer prevention study II, United States, 1982-1991," American Journal of Epidemiology, vol. 147, no. 7, pp. 652-659, 1998.

[36] D. Degnan, R. Harris, J. Ranney, D. Quade, J. A. Earp, and J. Gonzalez, "Measuring the use of mammography: two methods compared," American Journal of Public Health, vol. 82, no. 10, pp. 1386-1388, 1992.

[37] N. P. Gordon, R. A. Hiatt, and D. I. Lampert, "Concordance of self-reported data and medical record audit for six cancer screening procedures," Journal of the National Cancer Institute, vol. 85, no. 7, pp. 566-570, 1993. 


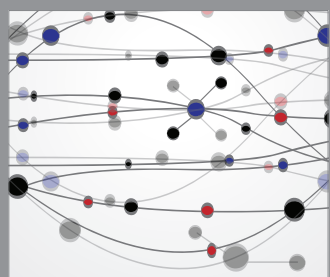

The Scientific World Journal
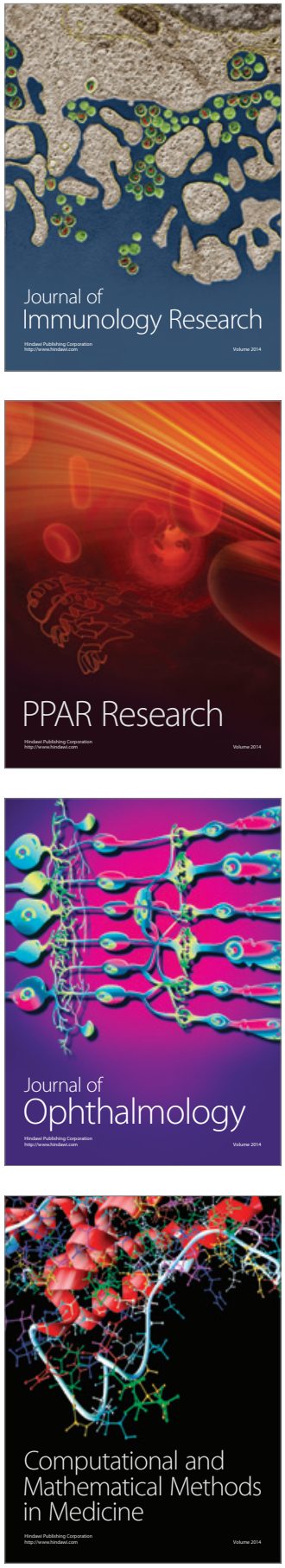

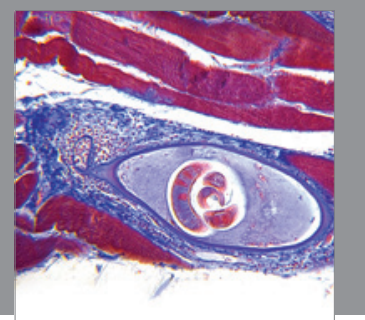

Gastroenterology

Research and Practice
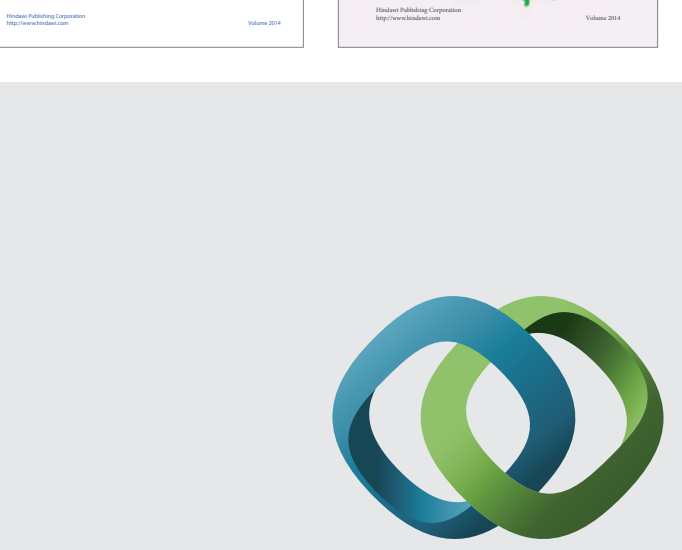

\section{Hindawi}

Submit your manuscripts at

http://www.hindawi.com
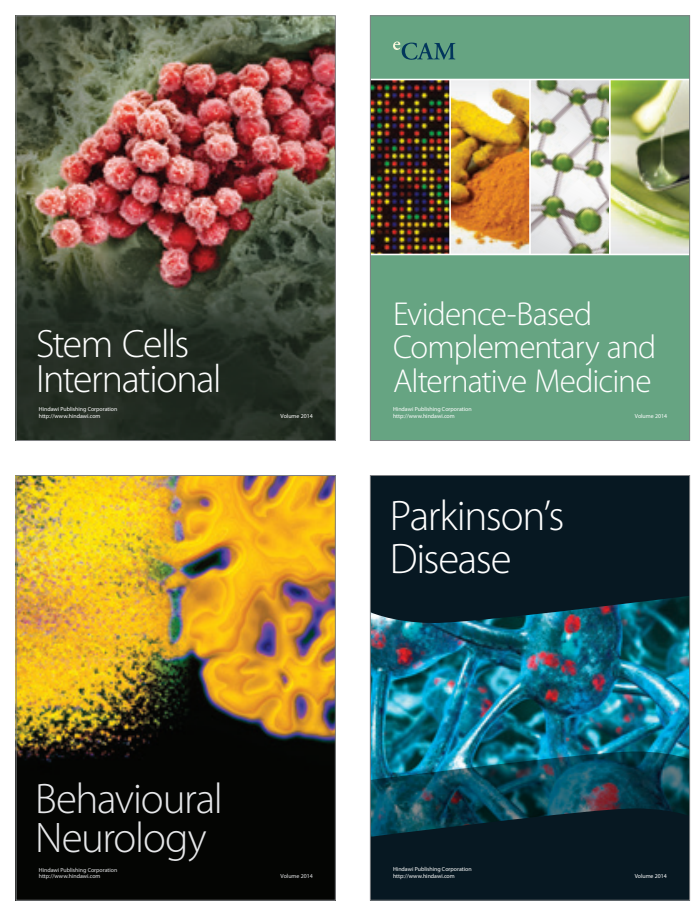

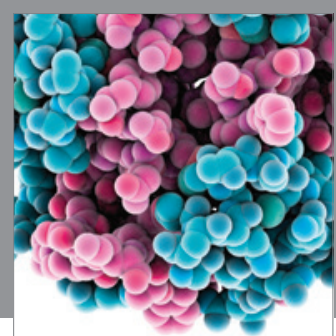

Journal of
Diabetes Research

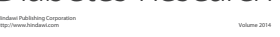

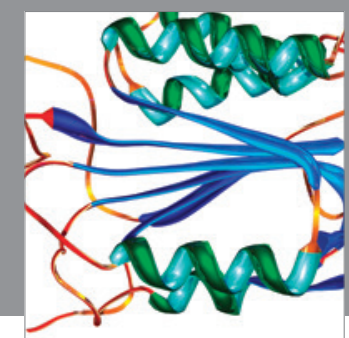

Disease Markers
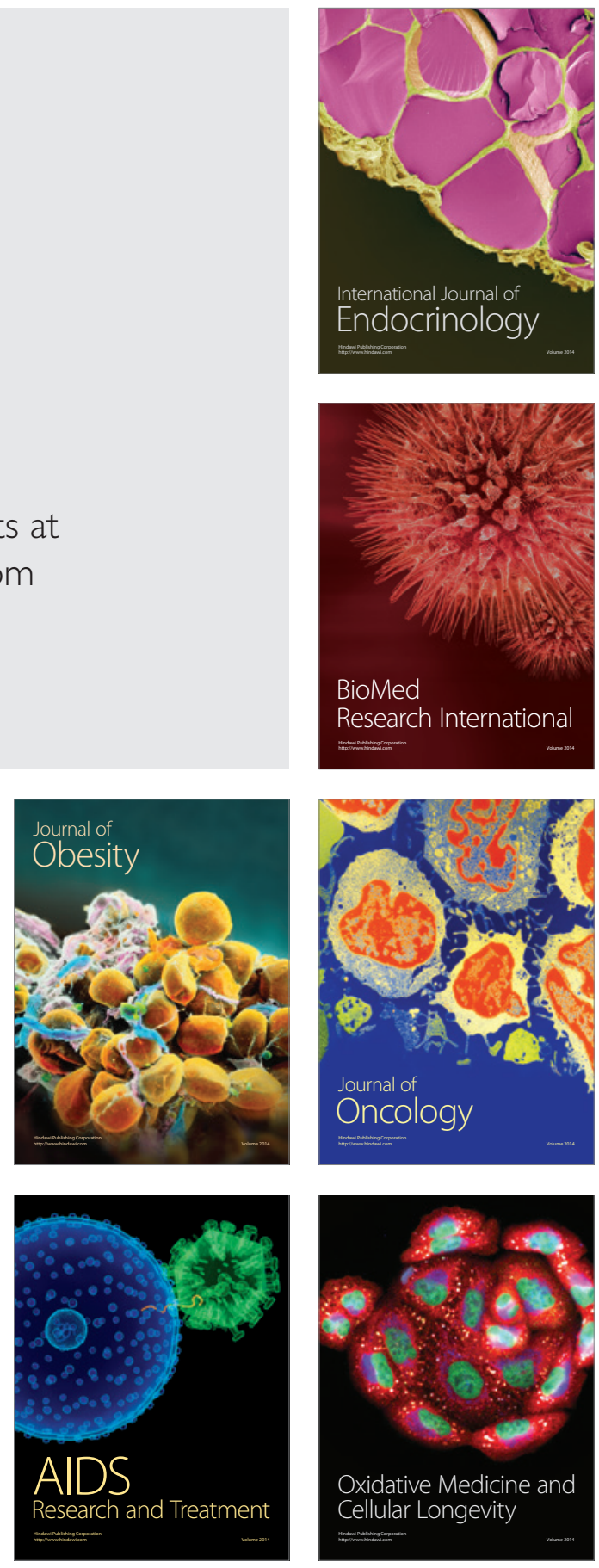